\title{
Efecto hipocolesterolémico del consumo de avena (Avena sativa) en un grupo de adultos de Chaclacayo, 2015
}

\author{
Hypocholesterolemic effect of consumption of oats (Avena sativa) in a group of adults Chaclacayo, \\ 2015 \\ Noelia Rodríguez Pérez*, Cinthia Díaz Caballero², Elisa Rodríguez López³
}

\begin{abstract}
RESUMEN
Objetivo: Determinar el efecto del consumo de avena (Avena sativa) sobre el nivel de colesterol y triglicéridos en suero. Metodología: Enfoque cuantitativo, de diseño pre experimental (pre y post) y de corte longitudinal. La muestra estuvo conformada por 26 personas adultas del asentamiento humano Nueva Alianza-Chaclacayo (Lima), de ambos sexos, entre 28 a 80 años, con niveles de colesterol > a 190mg/dl, quienes fueron seleccionados mediante muestreo no probabilístico de tipo intencional. El consumo fue de 60 g/día de avena en hojuelas por un periodo de 6 semanas. Resultados: Al término de la intervención se demostró disminución estadísticamente significativa en el nivel de colesterol $(\mathrm{T}=4,95 ; \mathrm{p}<0,05)$ de 217,04 mg/dl a 195,73 mg/dl, sin embargo, no hubo cambios estadísticamente significativos en el nivel de triglicéridos $(Z=-1,18 ; p>0,05)$. Conclusiones: El consumo de $60 \mathrm{~g}$ de avena en hojuelas durante un periodo de 6 semanas reduce el nivel de colesterol total.
\end{abstract}

Palabras clave: Avena sativa, colesterol total (CT), triglicéridos (TG).

\begin{abstract}
Objective: Determine the effect of the consumption of oats (Avena sativa) on the level of cholesterol and triglycerides in serum. Methodology: Quantitative approach, preexperimental design (pre and post) and longitudinal cut. The sample was formed by 26 adults of the Nueva Alianza-Chaclacayo, both enders, between 28 to 80 years old, with cholesterol levels $\geq$ a $190 \mathrm{mg} / \mathrm{dl}$, who were selected by sampling non-probability of intentional kind. The consumption was $60 \mathrm{~g}$ of oat flakes for 6 weeks. Results: Showed statistically significant reduction in cholesterol levels $(\mathrm{T}=4,95 ;<.05)$ of $217,04 \mathrm{mg} / \mathrm{dl}$ a $195,73 \mathrm{mg} / \mathrm{dl}$. However, there were no statistically significant changes in the level of triglycerides $(Z=-1,18 ; p>.05)$. Conclusion: In conclusion, the consumption of $60 \mathrm{~g}$ of oat flakes over a 6 week period significantly reduces the level of total cholesterol.
\end{abstract}

Keywords: Avena sativa, cholesterol total, triglycerides

\footnotetext{
${ }^{1}$ Licenciada en Nutrición Humana, Clínica Americana de Juliaca, Puno, Perú

${ }^{2}$ Licenciada en Nutrición Humana, EP Nutrición Humana, Facultad de Ciencias de la Salud, Universidad Peruana Unión, Lima, Perú

${ }^{3}$ Docente de EP Nutrición Humana, Facultad de Ciencias de la Salud, Universidad Peruana Unión, Lima, Perú
} 


\section{INTRODUCCIÓN}

Los lípidos como el colesterol y triglicéridos, cumplen funciones importantes en el organismo, sin embargo, debido a factores como la obesidad, dietas ricas en ácidos grasos saturados, inactividad física, alimentos refinados y causas genéticas, pueden elevarse en el plasma sanguíneo. De esta manera, cuando los niveles de colesterol se encuentran altos se diagnostica hipercolesterolemia y en el caso de los triglicéridos se denomina hipertrigliceridemia (Grundy, 2002). En consecuencia, constituyen un factor primordial para la generación de aterosclerosis y enfermedades cardiovasculares (ECV) (Díaz, Alarcón, \& Amaya, 2005; Segura, Agusti, \& Ruiz, 2013).

La Organización Mundial de la Salud (OMS) reportó que 4.4 millones de personas mueren como resultado de niveles de colesterol total elevados (OMS, 2016). Por otro lado, en Perú, la prevalencia de colesterol alto aumentó a 21.1\% en la población adulta mayor (INEI, 2014). De ahí que, la prevalencia de hiperlipidemia a nivel nacional revela un problema enfocado al ámbito de la salud pública ya que constituyen un riesgo para el desarrollo de las ECV y aumento de la mortalidad (Plaza, Villar, Mata, \& Pérez, 2000).

La National Colesterol Education Program Adult Treatment Panel (NCEP ATP III) recomienda que se debe aumentar la ingesta de fibra soluble para disminuir el colesterol sérico (Rubio, Moreno, \& Cabrerizo, 2004). Uno de los alimentos estudiados, rica en fibra soluble, es la avena que por su contenido de $\beta$-glucano puede reducir el nivel de colesterol total. Estudios recientes han demostrado el efecto de avena sobre el colesterol, como el estudio de Schuster, Benincá, Vitorazzi \& Morelo Dal Bosco (2015) quienes realizaron un estudio de caso y control, donde utilizaron harina de avena en sujetos de 22 a 60 años. El grupo control siguió una dieta habitual y el grupo experimental siguió una dieta habitual más 40 g de avena/día, durante 8 semanas, como resultado se obtuvo una reducción significativa del colesterol total en $19.66 \%$ y triglicéridos en $11.96 \%$. Otro estudio fue realizado por Ruiz, Rodríguez, Herrera y Cortés (2011) quienes determinaron el efecto de avena en pacientes con dislipidemia. Fue un estudio cuasi experimental realizado con personas entre edades de 20 y 60 años, quienes consumieron $60 \mathrm{~g}$ de avena licuada en agua durante tres meses. Los resultados evidenciaron una disminución estadísticamente significativa de CT de $48.8 \mathrm{mg} / \mathrm{dl}$ y sin embargo, los TG no mostraron cambios estadísticamente significativos.

La avena es un cereal perteneciente al reino Plantae de la familia de los Poaceae. Se afirma que la avena puede proceder del suroeste de Europa, del suroeste de Asia o incluso del norte de África, aunque las teorías más extendidas se inclinan por su origen en Asia Central (Carrera, 2005). Comprende alrededor de 70 especies y particularmente las más cultivadas son la Avena sativa L. y la Avena byzantina K. (Ramirez, 2007). En cuanto a la Avena sativa, nombre derivado del epíteto latín que significa "cultivada", es denominada comúnmente como avena blanca, avena común, avena cultivada o avena doméstica (Roger, 2003). La avena de forma integral no es apto para el consumo humano por lo que es procesada y como producto final se obtiene sémola, harina u hojuelas de avena (Soto, 2007). La avena en hojuelas por 100 gramos aporta 5.09 g de $\beta$-glucano(De-Sá, DeFrancisco y Soares, 1998).

El efecto hipocolesterolémico de la avena se debe a la fibra soluble denominado $\beta$-glucano el cual se ubica mayormente en las paredes celulares del endospermo y en la capa de aleurona. Los $\beta$-glucanos estructuralmente son homopolisacáridos lineales de glucosa unidos a través de enlaces $\beta-(1 \rightarrow 3)$ y $\beta-(1 \rightarrow 4)$ $\mathrm{y}$ que pueden presentar ramificaciones (Pizarro, Ronco y Gotteland, 2014). Por su característica de viscosidad tiende a disminuir la absorción del colesterol a nivel del intestino y del ácido bílico. Además, es fermentado por las bacterias intestinales que forman ácidos grasos de cadena corta (acetato, propionato y butirato) y al ser absorbidos inhiben la síntesis de colesterol hepático(Escudero y González, 2006).

Este estudio tiene como objetivo general determinar el efecto del consumo de avena (Avena sativa) sobre el nivel de colesterol y triglicéridos en suero de personas adultas del asentamiento humano Nueva Alianza-Chaclacayo, 2015. Como objetivos específicos comparar el nivel de colesterol total y triglicéridos en suero antes y después del consumo de avena, de la misma manera por edad y sexo.

\section{MATERIALES Y MÉTODOS}

Diseño y tipo de investigación. Estudio de enfoque cuantitativo, de diseño preexperimental (pre y post test) y de corte longitudinal (Hernández, Fernández \& Baptista, 2010)

Muestra. Estuvo conformada por 33 participantes voluntarios del asentamiento humano Nueva Alianza, de los cuales 4 abandonaron el estudio en la primera semana de tratamiento y 3 no se presentaron en la segunda evaluación de colesterol y triglicéridos, por 
lo que solo 26 participantes conformaron el estudio. La selección de la muestra se realizó mediante el muestreo no probabilístico de tipo intencional, porque las unidades de estudio no fueron escogidos aleatoriamente (Hernández et al., 2010). Estos fueron identificados mediante visitas domiciliarias de las familias del asentamiento consideradas según los criterios de inclusión. Se invitó a las personas que tuvieron diagnóstico de colesterol positivo o sospecha del mismo después de evaluar las muestras de sangre a 60 personas de los cuales 33 cumplieron los requisitos para el trabajo.

De los participantes se tuvo que el 34,6\% fueron adultos y 65,4\% adultos mayores. En cuanto al sexo, $61,5 \%$ fueron mujeres y 38,5\% varones. Por estado civil, el 61,5\% fueron casados y el 7,7\% separados o divorciados. Con respecto a nivel de educación el 57,7\% presenta educación secundaria seguida de educación primaria con 30,8\%. De acuerdo al lugar de procedencia 57,7\% procedían de la costa y 42,3\% de la sierra del Perú.

Criterios de inclusión y exclusión. Se incluyeron personas que viven en el asentamiento humano Nueva Alianza con nivel de colesterol $\geq$ a $190 \mathrm{mg} /$ dl, entre 25 y 80 años de edad y que firmaron el consentimiento para participar del proyecto. Se excluyeron personas que no participaron del segundo examen de colesterol y triglicéridos, que consumen medicamentos para el control del perfil lipídico, con diagnóstico de hipotiroidismo, enfermedad renal crónica o enfermedad hepática obstructiva. Personas que dejaron de consumir la avena menos de 38 días y aquellas que no culminaron el proceso de intervención.

Análisis bioquímico. Los participantes asistieron en ayunas por lo menos 12 horas antes de la toma de muestras de sangre. Se realizó la extracción de sangre en tubos de $5 \mathrm{ml}$, los que estaban debidamente codificados. Las muestras fueron analizadas por el laboratorio Global Consulting Corporation S.A.C. Se hizo la determinación de colesterol y triglicéridos utilizando el suero del tejido sanguíneo. El método fue enzimático-colorimétrico. Este procedimiento se aplicó al inicio y al finalizar el estudio.

Selección, racionamiento y administración de avena. Se seleccionó el tipo de avena en hojuelas marca "3 ositos” tomadas del lote 31-10-16B procedente de la fábrica Molitalia SA. La ración comprendió en $60 \mathrm{~g}$ de avena pesado en una balanza marca "Soehnle" para alimentos y empaquetado en bolsas de polietileno. Se distribuyeron siete paquetes una vez por semana en un periodo de 6 semanas. Se les indicó a los participantes que debían remojar la avena en una taza y media de agua por la noche y consumirlo en ayunas al día siguiente, esto durante 42 días o 6 semanas.

Proceso de recolección de datos. Se recolectaron los datos sociodemográficos, estado nutricional, valores de colesterol y triglicéridos en la Ficha del participante. Cada participante registró su consumo diario en la Ficha del control del consumo de avena lo que evidenció el cumplimiento por parte del participante. La información sobre los alimentos consumidos a largo plazo antes y durante la intervención se registró en la Ficha de Frecuencia de consumo de alimentos. La información sobre los alimentos consumidos el día inmediato anterior fue registrada en la Ficha del recordatorio de 24 horas. La información sobre las variables intervinientes se registró en la Ficha control del cumplimiento de hábitos saludables.

Procesamiento y análisis de datos. Los datos recolectados fueron procesados en el software estadístico IBM SPSS versión 22. Para el análisis descriptivo de las variables sociodemográficas, antropométricas, nivel de colesterol y triglicéridos se utilizó tablas de frecuencia. Para establecer diferencias significativas en las variables dieta $\mathrm{y}$ hábitos saludables se utilizó la prueba estadística de Friedman y Q-Cochran porque los datos son categóricos. En el análisis de la normalidad se utilizó la prueba estadística Shapiro Wilk debido al tamaño de la muestra $(<30)$. Para establecer diferencias significativas del variable colesterol total por edad y sexo se utilizó la prueba estadística T-Student, para los datos con distribución normal; y en la variable triglicéridos por edad y sexo, la prueba estadística Wilcoxon, cuando los datos no siguieron una distribución normal.

Consideraciones éticas. De acuerdo con los principios de respeto por la vida e integridad humana, se tomó en cuenta los siguientes criterios: No se han reportado efectos adversos sobre el consumo de $60 \mathrm{~g}$ de avena por lo que no se consideró de riesgo, brindar esta dosis a los participantes. La investigación se llevó a cabo bajo el consentimiento de los dirigentes de la población de estudio y de los participantes. En caso de ocurrir algún imprevisto, el participante tenía la opción de retirarse si así lo deseaba. A fin de proteger la privacidad de los participantes su identificación fue codificada y la información fue utilizada sólo para fines de la investigación. A todos los participantes se les dio a conocer el proceso y las condiciones de la investigación y como muestra de su aprobación se les hicieron firmar el consentimiento informado. 


\section{RESULTADOS}

Tabla 1

Diferencias del nivel de colesterol total antes y después del consumo de avena, según la prueba T-Student

\begin{tabular}{lllllllll}
\hline Colesterol & M & DS & Mín. & Máx. & Rango & T & gl & P \\
\hline Antes & 217,04 & 20,326 & 190 & 280 & 90 & 4,9 & 25 &, 00 \\
Después & 195,73 & 24,608 & 158 & 249 & 91 & 50 & & \\
\hline
\end{tabular}

En la tabla 1 se observa que existe diferencia estadísticamente significativa entre el antes $(\mathrm{M}=$ 217,04) y después $(M=195,73)$ de la intervención $(\mathrm{p}<0,05)$. Por lo tanto, el consumo de avena durante 6 semanas logró reducir el nivel de colesterol total de los participantes.

Tabla 2

Diferencias del nivel de colesterol total antes y después del consumo de avena por edad y sexo, según la prueba T-Student

\begin{tabular}{|c|c|c|c|c|c|c|c|c|c|}
\hline & $\begin{array}{l}\text { Colesterol } \\
\text { total }\end{array}$ & $\mathrm{M}$ & DS & Mín. & Máx. & Rango & $\mathrm{T}$ & $g l$ & $P$ \\
\hline \multicolumn{10}{|l|}{ Edad } \\
\hline \multirow{2}{*}{$\begin{array}{l}\text { Adulto } \\
\text { (28-59años) }\end{array}$} & Antes & 210,56 & 20,845 & 190 & 250 & 60 & 3,553 & 8 & ,007 \\
\hline & Después & 187,78 & 25,849 & 170 & 249 & 79 & & & \\
\hline \multirow{2}{*}{$\begin{array}{l}\text { Adulto mayor } \\
\text { (60-80años) }\end{array}$} & Antes & 220,47 & 19,803 & 192 & 280 & 88 & 3,558 & 16 & ,003 \\
\hline & Después & 199,94 & 23,615 & 178 & 248 & 90 & & & \\
\hline \multicolumn{10}{|l|}{ Sexo } \\
\hline \multirow[t]{2}{*}{ Femenino } & Antes & 215,06 & 21,736 & 190 & 280 & 90 & 3,790 & 15 & ,002 \\
\hline & Después & 195,94 & 20,863 & 170 & 232 & 61 & & & \\
\hline \multirow[t]{2}{*}{ Masculino } & Antes & 220,20 & 18,498 & 192 & 250 & 58 & 3,107 & 9 & ,013 \\
\hline & Después & 195,14 & 30,927 & 158 & 249 & 91 & & & \\
\hline
\end{tabular}

En la tabla 2 de acuerdo a la edad, se muestra que existe diferencia estadísticamente significativa entre el antes $(M=210,56)$ y después $(M=187,78)$ de la intervención $(\mathrm{p}<0,05)$ en la población de estudio. De la misma manera sucede en los adultos mayores $(p<0,05)$. Por ende, el consumo de avena en el periodo de 6 semanas logró reducir el colesterol total en los adultos y adultos mayores. Con respecto al sexo, se muestra que existe diferencia estadísticamente significativa entre el antes $(M=215,06)$ y después $(\mathrm{M}=195,94)$ de la intervención $(\mathrm{p}<0,05)$. De la misma manera sucede en el sexo masculino $(\mathrm{p}<0,05)$.

Tabla 3

Diferencias del nivel de triglicéridos antes y después del consumo de avena, según la prueba de Wilcoxon

\begin{tabular}{llllll}
\hline Triglicéridos & Media & Mediana & Desv. típ & Z & $p$ \\
\hline Antes & 200,31 & 181 & 82,915 & $-1,181$ &, 237 \\
Después & 171,38 & 166 & 35,842 & & \\
\hline
\end{tabular}

En la tabla 3 se observa que no existe diferencia estadísticamente significativa entre el antes (Mdn=181; DS=82,915) y después (Mdn=166;
$\mathrm{DS}=35,842)$ de la intervención ( $\mathrm{p}>0,05)$. Por tanto, el consumo de avena no fue efectivo en reducir el nivel de triglicéridos. 
Efecto hipocolesterolémico del consumo de avena (Avena sativa) en un grupo de adultos de Chaclacayo, 2015

Tabla 4

Diferencias del nivel de triglicéridos antes y después del consumo de avena por edad, según la prueba de Wilcoxon

\begin{tabular}{|c|c|c|c|c|c|c|}
\hline & Triglicéridos & Media & Mediana & Desv. típ & Z & $p$ \\
\hline \multicolumn{7}{|l|}{ Edad } \\
\hline \multirow{2}{*}{$\begin{array}{l}\text { Adulto } \\
\text { (28-59años) }\end{array}$} & Antes & 187,11 & 150,00 & 107,906 & $-0,593$ &, 553 \\
\hline & Después & 167,11 & 166,00 & 33,935 & & \\
\hline \multirow{2}{*}{$\begin{array}{l}\text { Adulto mayor } \\
\text { (60-80años) }\end{array}$} & Antes & 207,29 & 200,00 & 69,069 & $-1,539$ & ,124 \\
\hline & Después & 173,65 & 166,00 & 37,626 & & \\
\hline \multicolumn{7}{|l|}{ Sexo } \\
\hline \multirow[t]{2}{*}{ Femenino } & Antes & 185,69 & 153,50 & 91,549 & $-0,336$ & ,737 \\
\hline & Después & 170,13 & 168,50 & 34,415 & & \\
\hline \multirow[t]{2}{*}{ Masculino } & Antes & 223,70 & 218,50 & 64,345 & $-1,938$ &, 053 \\
\hline & Después & 173,40 & 163,00 & 39,839 & & \\
\hline
\end{tabular}

En la tabla 4 de acuerdo a la edad, se observa que tanto en adultos y adultos mayores no existe diferencia significativa entre el nivel de triglicéridos antes y después de la intervención en adultos y adultos mayores $(p>0,05)$.
Con respecto al sexo, se observa que no hubo diferencia estadísticamente significativa entre el nivel de triglicéridos antes y después de la intervención, tanto en el género femenino como en el masculino $(\mathrm{p}>0,05)$.

Tabla 5

Comparación de la frecuencia de consumo de alimentos reguladores antes y durante el consumo de avena

\begin{tabular}{ccccccc}
\hline \multirow{2}{*}{$\begin{array}{c}\text { Grupos de } \\
\text { alimentos }\end{array}$} & $\begin{array}{c}\text { Nivel de } \\
\text { frecuencia }\end{array}$ & \multicolumn{2}{c}{$\begin{array}{c}\text { Antes del consumo de } \\
\text { avena }\end{array}$} & \multicolumn{3}{c}{$\begin{array}{c}\text { Durante el consumo de } \\
\text { avena }\end{array}$} \\
\cline { 3 - 7 } & & $\mathbf{N}$ & $\%$ & $\mathbf{N}$ & $\%$ & $\mathrm{P}$ \\
\hline \multirow{2}{*}{ Frutas } & Bajo & 4 & 15,4 & 4 & 15,4 & $0,01^{*}$ \\
& Moderado & 21 & 80,8 & 12 & 46,2 & \\
& Alto & 1 & 3,8 & 10 & 38,5 & \\
Verduras & Bajo & 3 & 11,5 & 2 & 7,7 & 0,15 \\
& Moderado & 17 & 65,4 & 13 & 50 & \\
& Alto & 6 & 23,1 & 11 & 42,3 & \\
\hline
\end{tabular}

Nota: la comparación se hizo mediante las prueba estadística de Friedman ${ }^{*} p<0.05$

En la tabla 5 se muestra el consumo de alimentos reguladores. En el grupo de frutas se evidencia una diferencia significativa entre el antes y durante de la intervención $(\mathrm{p}<0,05)$. Es decir, aumentó la frecuencia de moderado a alto. Por el contrario el consumo del grupo de verduras se mantuvo sin cambios significativos ( $\mathrm{p}>0,05)$. 
Tabla 6

Distribución del estado nutricional según IMC

\begin{tabular}{ccccc}
\hline \multirow{2}{*}{ Estado nutricional } & \multicolumn{2}{c}{$\begin{array}{c}\text { Adulto } \\
\text { (28 a } 59 \text { años })\end{array}$} & \multicolumn{2}{c}{$\begin{array}{c}\text { Adulto mayor } \\
(60 \text { a 80 años }) \\
n=17\end{array}$} \\
\hline Delgadez & $n$ & $\%$ & $n$ & $\%$ \\
Normal & 0 & 0 & 1 & 5,9 \\
Sobrepeso & 1 & 11,1 & 10 & 58,8 \\
Obesidad I & 4 & 44,4 & 2 & 11,8 \\
Obesidad II & 3 & 33,3 & - & - \\
Obesidad & 1 & 11,1 & - & - \\
\hline
\end{tabular}

En la tabla 6 se muestra que el $44,4 \%$ de adultos se encontraban normales. presentaron sobrepeso y el $58,8 \%$ de adultos mayores

Tabla 7

Distribución del riesgo cardiovascular según el Índice cintura cadera (ICC)

\begin{tabular}{cccc}
\hline Edad & Riesgo de ECV & $\mathrm{n}$ & $\%$ \\
\hline Adulto (28-59años) & Riesgo moderado & 2 & 22,2 \\
& Riesgo muy alto & 7 & 77,8 \\
& Total & 9 & 100,0 \\
\hline Adulto mayor (60-80años) & Riesgo moderado & 6 & 35,3 \\
& Riesgo alto & 4 & 23,5 \\
& Riesgo muy alto & 7 & 41,2 \\
& Total & 17 & 100,0 \\
\hline
\end{tabular}

En la tabla 7 se muestra que el $77,8 \%$ de adultos presento riesgo muy alto de contraer ECV de acuerdo al índice cintura cadera. De la misma manera en los adultos mayores, el 41,2\% presentó riesgo muy alto de enfermedad cardiovascular (ECV).

\section{DISCUSIÓN}

El control del perfil lipídico es importante en la prevención de las enfermedades cardiovasculares, porque los niveles altos de colesterol y triglicéridos en suero favorecen la generación de arterosclerosis y enfermedad coronaria (González y García, 2013). Por otro lado, se ha reportado alimentos que regulan y normalizan las alteraciones del perfil lipídico (colesterol total y triglicéridos). Dentro de ello se tiene a la avena un cereal perteneciente a la familia Poaceae que a mediados de 1980 fue reconocida como un alimento saludable por su función cardioprotector (Sadiq-Butt, Tahir-Nadeem, Khan, y Shabir, 2008). Actualmente es difundido dentro de los alimentos peruanos según la tabla de composición de alimentos(CENAN, 2009). Los estudios reportan que el contenido de la fibra soluble ( $\beta$-glucanos) de la avena tiene un efecto hipocolesterolémico; por lo que lo convierte en un alimento alternativo para la prevención de enfermedades cardiovasculares (Wolever et al., 2011; Lo et al., 2011; Daou y Zhang, 2012).

El estudio evaluó el efecto del consumo de avena sobre los niveles de colesterol en personas adultas, encontrando que el promedio de colesterol sérico de los participantes antes de la intervención fue de 217,04 $\mathrm{mg} / \mathrm{dl}$ y después 195,73 mg/dl, es decir, se redujo en 21,31mg/dl. Esto demuestra que el consumo de $60 \mathrm{~g}$ de avena en hojuelas (Avena sativa) durante 6 semanas redujo significativamente el nivel de colesterol total. Sin embargo, uno de los factores que pudo haber influenciado en la disminución del colesterol, además seria el consumo de frutas, el cual se incrementó significativamente en los participantes (ver tabla 7). Se ha reportado que la fibra proveniente de frutas y vegetales se asocia inversamente a la disminución del colesterol, es decir por cada $10 \mathrm{~g}$ de consumo de fibra 
dietética reduce 12,5 mg/dl de colesterol total (Gontijo, Godoy, Gouvea, y Augusto, 2006).

Un estudio similar realizado por Ruiz et al. (2011), demostró que el consumo de $60 \mathrm{~g}$ de avena durante 12 semanas, también redujo significativamente el nivel de colesterol total en 48,8 mg/dl (de 240 a 198 $\mathrm{mg} /$ ). Cabe mencionar que ambas intervenciones tuvieron similar dosis pero tiempos de tratamiento diferentes. De igual manera, Schuster et al. (2015), determinaron el efecto del consumo de harina de avena sobre el perfil lipídico, durante 8 semanas en dos grupos de estudio: (1) grupo control con una dieta habitual y el (2) grupo experimental con dieta habitual de más de $40 \mathrm{~g}$ de avena. Los resultados mostraron una reducción del colesterol en 25,6 mg/ dl; no obstante, la cantidad de avena fue menor a los estudios anteriormente mencionados.

Uno de los componentes de la avena es la fibra -glucano que tiene la capacidad de disminuir los niveles de colesterol. Esta, al ingresar a la luz intestinal, interactúa con el colesterol y los ácidos biliares, impide la reabsorción de los lípidos y de esta manera ocasiona su eliminación por las heces. Adicionalmente, los $\beta$-glucano son fermentados por las bacterias intestinales formando ácidos grasos de cadena corta que son absorbidos y de esta manera inhiben la síntesis de colesterol hepático (Bello, 2000; Rondanelli, Opizzi y Monteferrario, 2009; Gunness et al., 2014). Tal como lo refiere Thongoun et al. (2013), en un estudio donde determinaron el efecto del consumo de $70 \mathrm{~g}$ de harina de avena ( $3 \mathrm{~g}$ de $\beta$-glucano) en personas hipercolesterolémicas obteniendo una reducción del colesterol total en un $5 \%(\mathrm{p}<0,05)$.

Mientras Charlton et al. (2012)at energy requirements for weight maintenance, probaron si $1,5 \mathrm{~g}$ /día de $\beta$-glucano en copos de avena era tan efectivo como $3 \mathrm{~g}$ de $\beta$-glucano en gachas de avena para disminuir el colesterol total. Después de la intervención se demostró una reducción significativa en cada grupo de intervención. De igual manera Biörklund, Rees, Mensink, y Onning (2005), utilizaron bebidas enriquecidas con $5 \mathrm{~g}$ de $\beta$--glucano de avena, lo que disminuyó significativamente en un $7,4 \%(\mathrm{p}<0,01)$ las concentraciones de colesterol total sérico. Los estudios concluyeron que la avena tiene su efecto hipocolesterolémico por su contenido de $\beta$-glucano. En este estudio se usó $60 \mathrm{~g}$ de avena en hojuelas que contiene aproximadamente de 2-3 g de $\beta$-glucano (De-Sá et al., 1998). Por consiguiente, se puede inferir que la reducción del colesterol total, se deba al efecto de los $\beta$-glucanos de la avena.

Al analizar el efecto del consumo de avena sobre el nivel de colesterol en personas adultas, según edad se encontró, en el grupo de adultos (28-59 años), una diferencia estadísticamente significativa $(p<0,05)$ en el promedio de colesterol sérico antes de la intervención de (210,56 mg/dl) y después (187,78 $\mathrm{mg} / \mathrm{dl}$ ) reduciendo en $22,78 \mathrm{mg} / \mathrm{dl}$ el colesterol total. Del mismo modo, en el grupo de adultos mayores se redujo en 20,53mg/dl (antes 220,47 mg/dl y después $199,94 \mathrm{mg} / \mathrm{dl})$.

A parte de ello, se añade que el 44,1\% de los adultos tenían sobrepeso, el 33,1\% presentó obesidad (ver tabla 6) y un 77,8\% tenían riesgo muy alto de desarrollar ECV (ver tabla 7). Cabe mencionar que el sobrepeso y la obesidad junto a la hipercolesterolemia constituyen factores de riesgo para la generación de arterosclerosis y eventos isquémicos(Loffredo et al., 2012). Además esta condición en la etapa adulta deteriora la calidad de vida en los años siguientes (la etapa del adulto mayor). Se menciona que el proceso de envejecimiento hace que el colesterol total aumente $2 \mathrm{mg} / \mathrm{dl}$ por año hasta los 65 años de edad y después desciende. Si no existe buenas condiciones de salud en la etapa adulta, una adecuada alimentación y estilo de vida adecuado, el adulto mayor estaría más propenso a la muerte (Hernández y Gonzáles, 2011; Gómez, 2005). Cabe resaltar que en este estudio se encontró que el $41,2 \%$ de los adultos mayores presentaron riesgo cardiovascular muy alto (ver tabla 7), por lo que era necesario una intervención para disminuir el riesgo de morbilidad.

Por lo tanto, el estudio demuestra el efecto hipocolesterolémico de la avena en la población adulta y adulta mayor, de manera que, podría ser una opción para incluir este alimento en la dieta de los adultos.

$\mathrm{Al}$ analizar el efecto del consumo de avena sobre el nivel de colesterol en personas adultas, según sexo, se encontró en el grupo femenino una diferencia estadísticamente significativa $(p<0,05)$ en el promedio de colesterol sérico antes de la intervención (215,06 mg/dl) y después (195,94 mg/dl). Lo cual indica una reducción de 19,12 $\mathrm{mg} / \mathrm{dl}$ del colesterol total. De igual manera ocurrió en el grupo masculino, con una diferencia estadísticamente significativa $(\mathrm{p}<0,05)$ de 25,06 mg/dl (antes 220,20 mg/ dl y después 195,14 mg/dl).

Una revisión de literatura refiere que las mujeres tienen menos colesterol total que los hombres en la etapa adulta, sin embargo las mujeres después de la menopausia pierden el nivel protector de los estrógenos. Esto promueve el aumento del colesterol y el riesgo de enfermedad coronaria (Hernández y Gonzáles, 2011). El estudio demostró un efecto reductor en ambos sexos, por lo que podría ser una opción que se incluya en la dieta de ambos grupos. 
Al evaluar la efectividad del consumo de avena en adultos, se encontró que el promedio de triglicéridos en suero antes de la intervención fue de $200,31 \mathrm{mg} /$ dl $(\mathrm{Mdn}=181)$ y después 171,38 mg/dl $(\mathrm{Mdn}=166)$; es decir, disminuyó en $28,93 \mathrm{mg} / \mathrm{dl}$ del dato inicial. Sin embargo, ésta variación no fue estadísticamente significativa, demostrando que el consumo de $60 \mathrm{~g}$ de avena en hojuelas (Avena sativa) durante 6 semanas no modifica los valores de triglicéridos significativamente en la muestra de estudio. Es necesario mencionar, que resultados similares fueron para la variable edad y sexo ( $p>0,05)$. De igual manera, Ruiz et al.(2011), demostraron que el consumo de avena no tuvo efecto significativo sobre los triglicéridos (de $256,57 \mathrm{mg}$ / dl a 237,42mg/dl). Asimismo, Alarcón et al. (2013), encontraron que el consumo de avena en pacientes con dislipidemia condujo a una variación de los niveles de triglicéridos de $260 \mathrm{mg} / \mathrm{dl}$ a $188 \mathrm{mg} / \mathrm{dl}$ pero sin diferencia significativa $(\mathrm{p}>0,05)$. Estas investigaciones corroboran los resultados, aunque se tomó el doble del tiempo para la intervención y dosis diferente para el segundo caso.

Por lo general, los estudios no reportan efectos del $\beta$-glucano sobre los triglicéridos. Se han experimentado sus efectos con diferentes dosis. Es así que, Charlton et al.(2012)at energy requirements for weight maintenance, encontraron que el consumo de 1,5 g/día de $\beta$-glucano en copos de avena y $3 \mathrm{~g} /$ día de $\beta$-glucano en gachas de avena, durante 6 semanas, no produjo cambios significativos en los niveles de triglicéridos $(p>0,05)$. Esto es corroborado por Reyna et al.(2006), quienes determinaron el efecto del consumo de $6 \mathrm{~g}$ de $\beta$-glucano de avena suplementado en la dieta del Paso II de la AHA en varones dislipidémicos, durante 8 semanas, no obteniendo cambios significativos en el nivel de triglicéridos.

Otras investigaciones realizadas en ratones de experimento, compararon el efecto del $\beta$-glucano de avena (fibra soluble) y frejoles negros (fibra insoluble), como resultado se encontró que no hubo cambios en el nivel de triglicéridos en ratones que consumieron avena. No obstante, sí hubo una reducción estadísticamente significativa en los ratones que consumieron frejoles negros (Morón, Infante, Ávila, García, y Liuzzi, 2010).

Sin embargo, otros estudios han demostrado que el consumo de Avena sativa, produce cambios sobre el nivel de triglicéridos. Lo confirma, Bautista, Calzada, Gutiérrez, y Mendoza (2012), en su estudio, donde demostraron que el consumo de $20 \mathrm{~g}$ de avena licuado con manzana redujo significativamente el nivel de triglicéridos en pacientes dislipidémicos (de 307,8 mg/dl a 193,6 mg/dl). Estos resultados podría deberse a la manzana acompañada en la avena, la que formo parte del tratamiento, y que por su alto contenido en fibra (pectina y celulosa), se obtuvieron resultados favorables. No obstante, en este estudio, hubo un aumento en el consumo de fruta por parte de los participantes, sin embargo, no se logró controlar la cantidad de ingesta diaria de frutas. También, Schuster et al. (2015), demostraron que el consumo de $40 \mathrm{~g}$ de avena en harina incluidos en la dieta habitual redujo los triglicéridos (de 147,92mg/dl a 137,39 mg dl) significativamente, en un periodo de 8 semanas.

Finalmente, existen factores intervinientes que podrían alterar los niveles de colesterol y triglicéridos, como las horas de sueño, consumo de alcohol, consumo de cigarrillo, el ejercicio físico, y consumo de alimentos. Estudios relacionan la carencia de horas de sueño nocturno con la disminución de las concentraciones de c-HDL(Ruiz, Rangel, Rodr, Rodr, y Rodr, 2014), de igual manera, el consumo excesivo de alcohol aumenta la síntesis de ácidos grasos, de colesterol y triglicéridos (Vivanco, 2014; Kim, Chu, Kim, y Moon, 2011), al mismo tiempo el hábito de fumar aumenta los niveles de colesterol total, c-LDL y disminuye el c-HDL (Rao, 2013; Sliwinska-Mossón, Mihulka, y Milnerowicz, 2014). Sin embargo, la práctica de ejercicio físico moderado reduce los niveles de triglicéridos y colesterol (Peñafiel y Guatemal, 2010; Ai-Lun, Shin-Da, Chia-Ting, JueLong, y Ko-Long, 2007). En el estudio estas variables no fueron controladas pero sí se las describieron. Al analizar si hubo variación entre los hábitos antes y durante la intervención se encontró que en los hábitos no hubo cambios significativos por parte de los participantes. De esta manera, se confirma la efectividad del consumo de avena sobre la reducción del colesterol sin la intervención significativa de los factores mencionados.

El consumo de 60g/día de avena (Avena sativa) por un periodo de 6 semanas redujo significativamente el nivel de colesterol total, del mismo modo, fue por edad y sexo. Sin embargo el consumo de avena por un periodo de 6 semanas no redujo significativamente el nivel de triglicéridos de los participantes, así mismo, fue por edad y sexo.

\section{Declaración de financiamiento y de conflicto de intereses:}

El estudio fue financiado por los autores, quienes declaran no tener algún tipo de conflicto de interés en la investigación realizada.

\section{Correspondencia:}

Noelia Rodríguez Pérez

Nutricionista Clínica. Clínica Americana de Juliaca. Jr. Loreto \#315 - La Rinconada, Juliaca, Perú e-mail: noeliarp.166@gmail.com 


\section{REFERENCIAS BIBLIOGRÁFICAS}

Ai-Lun, Y., Shin-Da, L., Chia-Ting, S., Jue-Long, W., \& Ko-Long, L. (2007). Effects of exercise intervention on patients with stroke with prior coronary artery disease: aerobic capacity, functional ability, and lipid profile: a pilot study. Journal of Rehabilitation Medicine, 39(1), 88-90. doi:10.2340/16501977-0021.

Alarcón, A., Buitrago, D., Romero, L., Sánchez, A. M., Onatra, W., \& Ríos, M. T. (2013). Efecto de la avena y/o lovastatina sobre el perfil lipídico en pacientes dislipidémicos del Hopital de Tunjuelito, Bogotá 20092008. Revista U.D.C.A. Actualidad Y Divulgación Cientifica, 16(2), 319-326.

Bautista, R., Calzada, D. J., Gutiérrez, M. de J., \& Mendoza, R. (2012). Efecto del licuado de avena con manzana sobre dislipidemias y sobrepeso - obesidad. Revista Electrónica de Investigación de Enfermeriía FESIUNAM, 1(2), 21-33.

Bello, J. (2000). Ciencia bromatológica: principios generales de los alimentos (p. 596). Madrid, España: Díaz de Santos.

Biörklund, M., Rees, A., Mensink, R. P., \& Onning, G. (2005). Changes in serum lipids and postprandial glucose and insulin concentrations after consumption of beverages with $\beta$-glucans from oats or barley: a randomised dosecontrolled trial. European Journal of Clinical Nutrition, 59(11), 1272-81. doi:10.1038/sj.ejcn.1602240.

Carrera, M. (2005). Prontuario de agricultura: Cultivos Agricolas (p. 940). Ministerio de Agricultura, Pesca y Alimentación , Secretaría General.

CENAN. (2009). Tablas peruanas de Composición de los alimentos (8 ed., p. 70). Lima: Instituto Nacional de Salud.

Charlton, K. E., Tapsell, L. C., Batterham, M. J., O'Shea, J., Thorne, R., Beck, E., \& Tosh, S. M. (2012). Effect of 6 weeks' consumption of $\beta$-glucan-rich oat products on cholesterol levels in mildly hypercholesterolaemic overweight adults. The British Journal of Nutrition, 107(7), 1037-47. doi:10.1017/S0007114511003850.

Daou, C., \&Zhang, H. (2012). OatB-Glucan: Its Role in Health Promotion and Prevention of Diseases. Comprehensive Reviews in Food Science and Food Safety, 11(4), 355365. doi:10.1111/j.1541-4337.2012.00189.x

De-Sá, R., De-Francisco, A., \& Soares, F. (1998). Concentração de B-glucanas nas diferentes etapas do procesamento da aveia (Avena sativa L.). Food Science and Technology (Campinas), 18(4), 1-8.

Díaz, M., Alarcón, D., \& Amaya, V. (2005). Efecto de un programa de atención nutricional sobre los valores del perfil lipidíco de trabajadores de la Pontificia Universidad Javeriana con diagnóstico de dislipidemia. Revista de La Facultad de Ciencias Universitas Scientiarum, 10, 71-80.

Escudero, E., \& González, P. (2006). La fibra dietética. Nutrición Hospitalaria, 21(2), 61-72.

Gómez, J. A. (2005). Dislipidemia en ancianos. Anales de La Facultad de Ciencias Médicas, 38(1-2). Retrieved from http://scielo.iics.una.py/scielo.php?pid=S181689492005000100007\&script=sci_arttext.
Gontijo, T., Godoy, S., Gouvea, S. R., \& Augusto, M. (2006). Association of dietary fiber with temporal changes in Serum cholesterol in japanese-brazilians. $J$ Nutr Sci Vitaminol, 52, 205-210.

González, J. P., \& García, R. J. (2013). Dislipidemias: controversias del riesgo residual. Rev Venez Endocrinol Metab, 11(2), 59-66.

Grundy, S. M. (2002). Nutrición y dieta en el tratamiento de la hiperlipidemia y arteriosclerosis. In M. Shils, J. Olson, M. Shike, \& C. Ross (Eds.), Nutrición en Salud y Enfermedad (9na ed., pp. 1377-1423). Mexico, D.F.: McGraw-Hill.

Gunness, P., Michiels, J., De Smet, S., Vanhaecke, L., Kravchuk, O., \& Gidley, M. J. (2014). Effects of oat $\beta$-glucan on bile salts diffusion across intestinal mucosa using the ussing chamber system. Journal of Nutrition \& Intermediary Metabolism, 1(2014), 45. doi:10.1016/j. jnim.2014.10.167.

Hernández, N. M., \& Gonzáles, W. (2011). Perfil lipídico y presión arterial en pacientes adultos que asistieron al laboratorio LV, Salud, R.L” Puerto Ordaz, Estado Bolivar. Universidad de Oriente Núcleo Bolívar Escuela de Ciencias de la Salud.

Hernández, R., Fernández, C., \& Baptista, M. del P. (2010). Metodología de la Investigación. (J. Mares, Ed.) (5ºd., p. 656). Buenos Aires: McGraw-Hill.

INEI. (2014). Estado de la Población Peruana (pp. 1-48). Lima.

Kim, J., Chu, S., Kim, K., \& Moon, J.-R. (2011). Alcohol use behaviors and risk of metabolic syndrome in South Korean middle-aged men. BMC Public Health, 11(489), 1-9.

Lo, Y., Yang-chao Luo, Chen, G., Zhen-lei, X., Wang, Q., Zhao, L., \& Bao-ping, J. (2011). Comparison of Protective Effects Between Oat B-Glucan and PhenolRich Extracts in Hyperiipidemic iCR $\mathrm{i} \backslash$ 1ice. Journal of Food and Drug Analysis, 19(I), 49-57.

Loffredo, L., Martino, F., Carnevale, R., Pignatelli, P., Catasca, E., Perri, L., ... Violi, F. (2012). Obesity and hypercholesterolemia are associated with NOX2 generated oxidative stress and arterial dysfunction. The Journal Of Pediatrics, 161(6), 1004-9.

Morón, M. C., Infante, B., Ávila, A., García, O. E., \& Liuzzi, J. P. (2010). Efecto del consumo de dietas con avena y caraotas negras sobre el perfil lipídico en un modelo experimental en rata. Revista Del Instituto de Higiene “Rafael Rangel”, 41(1), 31-37.

OMS. (2016). Panorama General. Prevención de las enfermedades crónicas: una inversión vital (p. 34). Canadá. Retrieved from http://www.who.int/chp/ chronic_disease_report/overview_sp.pdf?ua=1

Peñafiel, D., \& Guatemal, W. (2010). Prevalencia de dislipidemias y sus factores de riesgo en adultos que acuden al centro de Salud $N^{\circ} 1$ de la ciudad de Ibarra, provincia de Imbabura. Octubre del 2009-diciembre del 2010. Universidad Tecnica del Norte. 
Pizarro, S., Ronco, A. M., \& Gotteland, M. (2014). $\beta$-glucanos: i qué tipos existen y cuáles son sus beneficios en la salud? Revista Chilena de Nutrición, 41(3), 439-446.

Plaza, I., Villar, F., Mata, P., \& Pérez, F. (2000). Control de la colesterolemia en España , 2000 . Un instrumento para la prevención cardiovascular. Rev Esp Cardiol., 53(6), 815-837.

Ramirez, S. (2007). Efecto del sistema de siembra y estado de madurez sobre producción y calidad nutricional de siete variedades de avena (Avena sativa). Universidad Autónoma de Chihuahua.

Rao, C. S. (2013). The effect of chronic tabacco smoking and chewing on the lipid profile. Journal of Clinical and Diagnostic Research, 7(1), 31-34.

Reyna, N., Bermúdez, V., Mengual, E., Arias, N., Cano, C., Leal, E., ... Inglett, G. E. (2006). Oat derived-glucan significantly improves HDLc and diminishes LDLc and Non-HDL cholesterol in overweigh individuals with mild hypercholesterolemia. Revista Latinoamericana de Hipertensión, 1(3), 28-34.

Roger, J. D. P. (2003). Salud por los alimentos (p. 383). Buenos Aires: ACES.

Rondanelli, M., Opizzi, A., \& Monteferrario, F. (2009). The biological activity of $\beta$-glucans. Minerva Medica, 100(3), 237-245.

Rubio, M. A., Moreno, C., \& Cabrerizo, L. (2004). Guías para el tratamiento de las dislipemias en el adulto: Adult Treatment Panel III ( ATP-III ). Endocrinología y Nutrición, 51(5), 254-265.

Ruiz, E. E., Rodríguez, O., Herrera, A., \& Cortés, J. C. (2011). Consumo de avena (Avena sativa) y prevención sin restricción dietética. Aten Fam, 18(2), 35-37.

Ruiz, N., Rangel, A., Rodr, C., Rodr, L., \& Rodr, V. (2014). Relación entre el déficit de sueño nocturno, el exceso de peso y las alteraciones metabólicas en adolescentes. Arch Argent Pediatr, 112(6), 511-518.
Sadiq-Butt, M., Tahir-Nadeem, M., Khan, M. K. I., \& Shabir, R. (2008). Oat : unique among the cereals. Eur J Nutr, 47(1), 68-79. doi:10.1007/s00394-008-0698-7.

Schuster, J., Benincá, G., Vitorazzi, R., \& Morelo Dal Bosco, S. (2015). Effects of oats on lipid profile, insulin resistance and weight loss. Nutricion Hospitalaria, 32(5), 2111-2116. doi:10.3305/nh.2015.32.5.9590.

Segura, L., Agusti, R., \& Ruiz, E. (2013). Factores de Riesgo de las Enfermedades Cardiovasculares en el Perú II . Estudio TORNASOL II comparado con TORNASOL I después de cinco años. Revista Peruana de Cardiología, XXXIX(1), 55.

Sliwinska-Mossón, M., Mihulka, E., \& Milnerowicz, H. (2014). Assessment of lipid profile in non-smoking and smoking young health persons. PubMed, 71(11), 58587.

Soto, M. A. (2007). Estudio del Efecto del Espesor de laminado en un cereal de avena con almidón retrogrado sobre su índice glicémico. Universidad Austral de Chile.

Thongoun, P., Pavadhgul, P., Bumrungpert, A., Satitvipawee, P., Harjani, Y., \& Kurilich, A. (2013). Effect of oat consumption on lipid profiles in hypercholesterolemic adults. Journal of the Medical Association of Thailand, 96(5), 25-32.

Vivanco, J. A. (2014). Prevalencia y factores asociados a dislipidemia en pacientes 18 y 64 años. Hospital Vicente Corral Moscoso, 2013. Universidad de Cuenca.

Wolever, T. M. S., Gibbs, A. L., Brand-miller, J., Duncan, A. M., Hart, V., Lamarche, B., ... Duss, R. (2011). Bioactive oat B-glucan reduces LDL cholesterol in Caucasians and non-Caucasians. Nutritions Journal, 10(130), 1-5.

Recibido: 28/02/16

Aceptado: 06/05/16 